\title{
Retinal nerve fiber layer evaluation in multiple sclerosis with spectral domain optical coherence tomography
}

\author{
Aziz A Khanifar' \\ George J Parlitsis' \\ Joshua R Ehrlich' \\ Grant D Aaker' \\ Donald J D'Amico' \\ Susan A Gauthier ${ }^{2}$ \\ Szilárd Kiss'
}

'Departments of Ophthalmology, ${ }^{2}$ Neurology, Judith Jaffe Multiple

Sclerosis Center, Weill Cornell

Medical College, New York, NY, USA
This article was published in the following Dove Press journal:

Clinical Ophthalmology

13 September 2010

Number of times this article has been viewed

Purpose: Histopathologic studies have reported retinal nerve fiber layer (RNFL) thinning in various neurodegenerative diseases. Attempts to quantify this loss in vivo have relied on time-domain optical coherence tomography (TDOCT), which has low resolution and requires substantial interpolation of data for volume measurements. We hypothesized that the significantly higher resolution of spectral-domain optical coherence tomography (SDOCT) would better detect RNFL changes in patients with multiple sclerosis, and that RNFL thickness differences between eyes with and without optic neuritis might be identified more accurately.

Methods: In this retrospective case series, patients with multiple sclerosis were recruited from the Judith Jaffe Multiple Sclerosis Center at Weill Cornell Medical College in New York. Patients with a recent clinical diagnosis of optic neuritis (less than three months) were excluded. Eyes with a history of glaucoma, optic neuropathy (other than multiple sclerosis-related optic neuritis), age-related macular degeneration, or other relevant retinal and/or optic nerve disease were excluded. Both eyes of each patient were imaged with the Heidelberg Spectralis ${ }^{\circledR}$ HRA + OCT. RNFL and macular thickness were measured for each eye using the Heidelberg OCT software. These measurements were compared with validated published normal values, and were modeled as linear functions of duration of disease. The odds of an optic neuritis diagnosis as a function of RNFL and macular thickness were calculated.

Results: Ninety-four eyes were prospectively evaluated using OCT. Ages of patients ranged from 26 to 69 years, with an average age of 39 years. Peripapillary RNFL thinning was demonstrated in multiple sclerosis patients; mean RNFL thickness was $88.5 \mu \mathrm{m}$ for individuals with multiple sclerosis compared with a reported normal value of $97 \mu \mathrm{m}(P<0.001)$. Eyes with a history of optic neuritis had more thinning compared with those without optic neuritis $(83.0 \mu \mathrm{m}$ versus $90.5 \mu \mathrm{m}$, respectively, $P=0.02$ ). No significant differences were observed in macular thickness measurements between eyes with and without optic neuritis, nor were macular thickness measurements significantly different from normal values. As a function of multiple sclerosis duration and controlling for age, RNFL thickness was decreased in patients with a duration of multiple sclerosis greater than five years compared with those with a duration less than or equal to one year $(P=0.008)$.

Conclusions: Patients with a history of multiple sclerosis had RNFL thinning that was detectable on SDOCT. Decreasing RNFL thickness in eyes with optic neuritis was found, and the odds of having optic neuritis were increased significantly with decreasing RNFL thickness. Average RNFL thinning with increasing duration of disease was an excellent predictor of a reported history of optic neuritis. SDOCT retinal imaging may represent a high-resolution, objective, noninvasive, and easily quantifiable in vivo biomarker of the presence of optic neuritis and severity of multiple sclerosis.

Keywords: multiple sclerosis, spectral-domain optical coherence tomography, optical coherence tomography, nerve fiber layer, nerve fiber layer thickness, optic neuritis 


\section{Introduction}

Neurodegenerative diseases such as multiple sclerosis, Alzheimer disease, and Parkinson's disease, are characterized by axonal lesions throughout the central nervous system, including the eye. ${ }^{1}$ Specifically with multiple sclerosis, histopathologic studies have reported retinal nerve fiber layer (RNFL) thinning, and these changes have been correlated with disease activity and white matter lesion volume on neuroradiologic studies, such as magnetic resonance imaging. ${ }^{2-5}$ The ease of in vivo cross-sectional retinal tissue imaging with optical coherence tomography (OCT) facilitates the use of the retina as a surrogate for multiple sclerosis disease activity. Prior attempts to quantify RNFL loss and to monitor multiple sclerosis patients have almost exclusively been with time-domain OCT (TDOCT). ${ }^{1,6-21}$

In addition to having a significantly lower axial resolution, TDOCT has limited image acquisition, retinal segmentation, and precise long-term monitoring capabilities. On the other hand, spectral-domain OCT (SDOCT), the newest iteration of OCT technology, is characterized by markedly increased image acquisition speed and improved axial resolution, among other features, and has been increasingly utilized for evaluation of a variety of ocular diseases, such as glaucoma, age-related macular degeneration, macular hole, epiretinal membrane, and diabetic macular edema. RNFL thickness measurement with this technology has been reported to be more reliable and reproducible compared with TDOCT. ${ }^{22-24}$ Exploitation of SDOCT's features and advantages compared with TDOCT could greatly improve and expand the means of detection and monitoring of multiple sclerosis disease activity.

We hypothesize that SDOCT imaging of the retina in patients with multiple sclerosis may provide a highresolution, objective, noninvasive, and easily quantifiable in vivo biomarker of severity of disease and presence of optic neuritis. To the best of our knowledge, this report represents one of the first such assessments of the retina in multiple sclerosis patients specifically with high-resolution SDOCT. ${ }^{25,26}$

\section{Methods}

This was a retrospective case series approved by the Weill Cornell Medical College Institutional Review Board. Subjects were recruited from the Judith Jaffe Multiple Sclerosis Center, Weill Cornell Medical College, Department of Neurology, in New York. Optic nerve and macular imaging was performed in the Department of Ophthalmology at Weill Cornell Medical College. Subjects with a diagnosis of multiple sclerosis, with or without a history of optic neuritis, were included. Eyes with a history of glaucoma, optic neuropathy (other than multiple sclerosis-related optic neuritis), age-related macular degeneration, or other relevant retinal or optic nerve diseases were excluded. Patients with a recent history of optic neuritis (less than three months' duration) were also excluded. Both eyes of each patient were imaged with the high-resolution Heidelberg Spectralis ${ }^{\circledR}$ HRA + OCT (Heidelberg Engineering, Germany) by a trained operator (GJP, GDA, AAK, or SK). Scanning protocols included both a circular $3.4 \mathrm{~mm}$ scan centered on the optic nerve head and a volumetric scan of the macula centered on the fovea (73 horizontal B-scans covering a superior-to-inferior distance of $4.6 \mathrm{~mm}$ ). In a setting separate from image acquisition, a masked observer excluded poor quality images from the final analysis (AAK). RNFL and macular thickness were measured for each eye using the Heidelberg software. To ensure improved accuracy in both RNFL and macular thickness measurements, each scan was individually reviewed, and appropriate adjustments of the segmentation lines were made in a masked fashion. RNFL measurements were reported by sector, ie, temporal, superotemporal, superonasal, nasal, inferonasal, inferotemporal, and average (see Figure 1). These RNFL measurements were compared with the Heidelberg normative database. Macular thickness was reported in a modified ETDRS (Early Treatment of Diabetic Retinopathy Study) macular map with the central subfield $1.00 \mathrm{~mm}$ in diameter, and the inner and outer subfields having diameters of $2.22 \mathrm{~mm}$ and $3.45 \mathrm{~mm}$, respectively.

Summary statistics, including age, gender, and duration of disease were compared between the subjects with optic neuritis (in either or both eyes) and those without optic neuritis (in either eye) using the Mann-Whitney $U$ test for differences in age and duration of disease, and Fisher's exact test for differences in gender distribution between groups.

The normality of the data distributions for RNFL and macular thickness measurements were checked with the Shapiro-Wilk test. Sample $t$-tests were conducted to compare average RNFL and macular thickness measurements of multiple sclerosis patients and published normal measurements. RNFL thicknesses were compared with the 50th percentile values provided in the validated Heidelberg software. Macular thickness measurements were compared with published normative values. ${ }^{27}$

To determine the relative chance of a diagnosis of optic neuritis given an eye's decreasing RNFL or macular thickness, odds ratios (ORs) were calculated. This was done by 

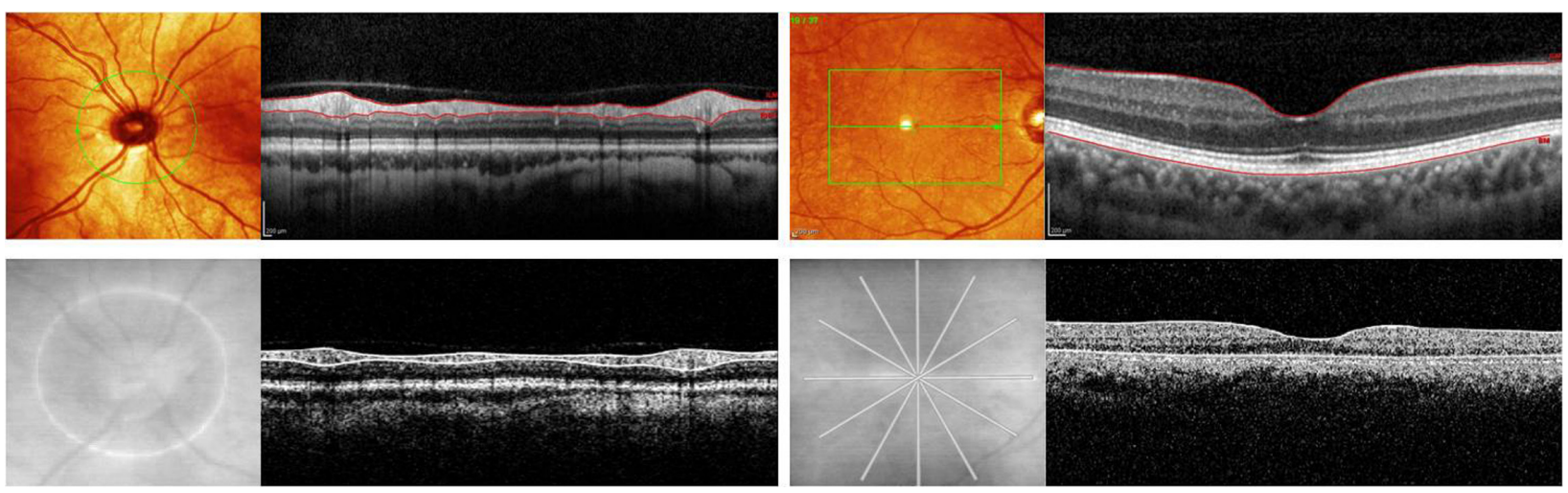

Figure I Comparative time-domain and spectral-domain OCT scans of peripapillary RNFL and macula in subjects with multiple sclerosis. In all four panels, OCT B-scans are accompanied by the corresponding fundus images indicating B-scan locations. Upper left: Heidelberg Spectralis ${ }^{\circledR}$ HRA + OCT Circular B-Scan. Red lines represent inner and outer boundaries of the RNFL. Upper right: Heidelberg Spectralis ${ }^{\circledR}$ HRA + OCT macular thickness scan composed of 73 horizontal B-scans within the green box area. Top red line represents the vitreoretinal interface (ie, inner boundary of macular thickness measurement). Bottom red line represents retinal pigment epithelium-choroid interface (ie, outer boundary of macular thickness measurement). Lower left: Stratus OCT Circular B-Scan. White lines represent inner and outer boundaries of RNFL. Lower right: Stratus OCT macular thickness scan composed of six radial B-scans. Top white line represents the vitreoretinal interface, and bottom white line represents the inner segment-outer segment interface.

Abbreviations: OCT, optical coherence tomography; RNFL, retinal nerve fiber layer.

using generalized estimating equations while adjusting for age and correlation between the right and left eyes of the same patient. Generalized estimating equations were also used to model RNFL and macular thickness as a function of duration of disease, while adjusting for age and intereye correlation. Semirobust variance estimation (Huber-White sandwich estimator) was used to estimate the standard error of parameters, ${ }^{28}$ and model selection was guided by the quasilikelihood under the independence model criterion method. ${ }^{29}$ Analyses were performed using Stata/IC 11.0 (StataCorp, College Station, TX). All statistical tests were two-sided with a level of significance of 0.05 .

\section{Results}

Peripapillary and macular OCT imaging was performed on 94 eyes of patients with multiple sclerosis. No images were excluded from the final analysis due to poor image quality. Patient age ranged from 26 to 69 years (median 39 years). The median ages of patients with and without optic neuritis were 40 and 33 years, respectively. The percentage of subjects who were female was $68.1 \%$, and for females with and without optic neuritis the percentages were $84.0 \%$ and $62.3 \%$, respectively. Median disease duration for all subjects with multiple sclerosis was 30.9 months, and for subjects with and without optic neuritis, median disease duration was 54.0 and 30.3 months, respectively. No significant differences with regard to age, gender, or disease duration were found between eyes with and without optic neuritis.

Peripapillary RNFL thinning was demonstrated (Table 1); mean thickness was $88.5 \mu \mathrm{m}(P<0.001)$ for individuals with multiple sclerosis when compared with eyes of patients without multiple sclerosis (normals, $97 \mu \mathrm{m}$ ). Eyes with a history of optic neuritis had thinner RNFL measurements than those without a history of optic neuritis $(83.0 \mu \mathrm{m}$ versus $90.5 \mu \mathrm{m}$, respectively, $P=0.02$ ).

Five subjects declined to have macular scanning performed, therefore macular thickness was measured in only 84 of 94 eyes (Table 1). The mean central subfield thickness for all eyes was $270.9 \mu \mathrm{m}$, compared with $270.2 \mu \mathrm{m}$ for Heidelberg device-specific published normative data $(P>0.05) .{ }^{27}$ Macular thinning in the inner nasal, outer nasal, and outer inferior sectors of all multiple sclerosis subjects when compared with controls was noted $(331.2 \mu \mathrm{m}$, $P=0.03$; $331.9 \mu \mathrm{m}, P<0.001$; and $320.2 \mu \mathrm{m}, P=0.004$, respectively).

Decreasing RNFL thickness measurements were associated with an increased risk of having a diagnosis of optic neuritis in that eye (Table 2). For example, a $5 \mu \mathrm{m}$ decrease in average RNFL thickness was likely to increase the odds of having a diagnosis of optic neuritis (OR 1.33, 95\% confidence interval [CI] 1.08-1.67, $P=0.009)$.

Increased average RNFL thinning with increased duration of disease was demonstrated (Table 3). The average RNFL thickness for subjects with disease duration greater than 60 months was less than that measured in subjects with a less than 13-month disease duration $(P=0.008)$. However, there was no significant difference in average RNFL thickness for those with 13-60 months' disease duration compared with those with less than 13 months' disease duration $(P=0.25)$. Macular thickness 
Table I Mean RNFL thickness measurements (top, $\mu \mathrm{m}$ ) and mean macular thickness measurements (bottom, $\mu \mathrm{m}$ ) with the Heidelberg Spectralis ${ }^{\circledR}$ HRA + OCT in patients with MS. Mean RNFL thickness for all eyes compared with normative data.

\begin{tabular}{|c|c|c|c|c|c|c|}
\hline RNFL & Normal* $^{*}$ & All MS (94) & $P$ & Non-ON (69) & ON (25) & $P$ \\
\hline Temporal & 74 & $62.6 \pm 15.2$ & $<0.001$ & $65.2 \pm 4.4$ & $55.5 \pm 15.5$ & 0.006 \\
\hline Superior-temporal & 133 & $119.8 \pm 23.7$ & $<0.001$ & $123.0 \pm 23.3$ & $110.9 \pm 23.1$ & 0.03 \\
\hline Superior-nasal & 105 & $96.1 \pm 26.1$ & 0.001 & $96.8 \pm 26.1$ & $94.5 \pm 26.4$ & 0.71 \\
\hline Nasal & 72 & $65.7 \pm 17.5$ & $<0.001$ & $66.8 \pm 18.1$ & $62.8 \pm 15.8$ & 0.33 \\
\hline Inferior-nasal & 107 & $103.8 \pm 26.2$ & 0.23 & $105.0 \pm 25.0$ & $100.4 \pm 29.6$ & 0.45 \\
\hline Inferior-temporal & 140 & $130.4 \pm 21.3$ & $<0.001$ & $134.2 \pm 21.0$ & $119.9 \pm 18.9$ & 0.004 \\
\hline Superior & - & $107.9 \pm 21.4$ & - & $109.8 \pm 21.7$ & $102.7 \pm 20.1$ & 0.16 \\
\hline Inferior & - & $117.0 \pm 18.8$ & - & $119.3 \pm 17.9$ & $110.5 \pm 20.1$ & 0.04 \\
\hline Average & 97 & $88.5 \pm 13.8$ & $<0.001$ & $90.5 \pm 13.2$ & $83.0 \pm 14.0$ & 0.02 \\
\hline Macula & Normal $^{\dagger}$ & All MS (84) & $P$ & Non-ON (59) & ON (25) & $P$ \\
\hline Inner temporal & 322.6 & $323.1 \pm 14.5$ & 0.76 & $325.3 \pm 14.5$ & $317.8 \pm 13.1$ & 0.02 \\
\hline Inner superior & 336.0 & $332.7 \pm 17.0$ & 0.08 & $335.2 \pm 17.5$ & $327.0 \pm 14.6$ & 0.05 \\
\hline Inner nasal & 335.0 & $331.2 \pm 16.3$ & 0.03 & $332.9 \pm 17.1$ & $327.2 \pm 13.9$ & 0.14 \\
\hline Inner inferior & 334.9 & $334.0 \pm 16.1$ & 0.62 & $336.3 \pm 16.3$ & $328.6 \pm 14.4$ & 0.04 \\
\hline Outer temporal & 320.1 & $317.6 \pm 15.2$ & 0.14 & $319.0 \pm 14.2$ & $314.3 \pm 17.0$ & 0.19 \\
\hline Outer superior & 329.6 & $327.8 \pm 18.9$ & 0.38 & $329.6 \pm 18.9$ & $323.6 \pm 18.4$ & 0.19 \\
\hline Outer nasal & 339.5 & $331.9 \pm 18.0$ & $<0.001$ & $334.3 \pm 17.8$ & $326.3 \pm 17.6$ & 0.06 \\
\hline Outer inferior & 325.4 & $320.2 \pm 16.2$ & 0.004 & $322.4 \pm 15.5$ & $314.9 \pm 16.9$ & 0.05 \\
\hline Central subfield & 270.2 & $270.9 \pm 17.3$ & 0.72 & $273.0 \pm 18.0$ & $265.9 \pm 14.4$ & 0.08 \\
\hline
\end{tabular}

Note: *Mean macular thickness for all eyes compared with published controls. Thickness measurements from eyes without ON are compared with those of eyes with ON. Eyes affected by MS show statistically significant RNFL thinning compared with normals. MS subjects with a history of OP have an even greater thinning than those without a history of ON. MS subjects have inner nasal, outer nasal, and outer inferior macular thinning compared with published normals. ${ }^{27} \dagger$

Abbreviations: MS, multiple sclerosis; OCT, optical coherence tomography; ON, optic neuritis; RNFL, retinal nerve fiber layer.

measurements were not observed to vary as a function of disease duration.

\section{Discussion}

To our knowledge, this study is one of the first to utilize high-resolution SDOCT to study retinal changes in patients with multiple sclerosis. ${ }^{25,26}$ Utilizing the Heidelberg Spectralis ${ }^{\circledR}$ HRA + OCT, we were able to demonstrate significant RNFL thinning in multiple sclerosis patients compared with controls. As well as in eyes with optic neuritis compared with those without optic neuritis. In general, prior studies with low-resolution TDOCT are consistent with our findings using SDOCT.

Kerrison et al, using histopathologic analysis, demonstrated a loss of RNFL thickness in the temporal quadrant in multiple sclerosis-affected eyes. ${ }^{30}$ Parisi et al, using an early iteration of TDOCT technology, demonstrated reduced temporal and overall RNFL thickness in eyes with and without optic neuritis when compared with controls. ${ }^{18}$ Fisher et al, using the Stratus TDOCT, similarly detected a reduction in RNFL thickness in subjects with multiple sclerosis, in addition to demonstrating an RNFL thickness difference between eyes with and without optic neuritis. ${ }^{16}$ Costello et al, using the Stratus TDOCT device, demonstrated a difference in RNFL thickness between eyes with and without optic neuritis. ${ }^{15}$
Finally, Trip et al showed a difference in RNFL thickness between optic neuritis-affected eyes and controls as well as between optic neuritis-affected eyes and optic neuritisunaffected eyes, however, no difference was detected between optic neuritis-unaffected eyes and controls. ${ }^{20}$ Other investigators utilizing SDOCT demonstrated significant RNFL thinning in eyes of patients with multiple sclerosis compared with healthy eyes, but they found differences in all four quadrants in addition to average thickness. However, they did not evaluate differences between eyes with and without optic neuritis, nor did they evaluate macular thickness measurements in these subjects. ${ }^{25}$

In the current study, patients with multiple sclerosis had significantly thinner inner nasal and outer nasal maculas than normal, and those eyes affected by optic neuritis had significant macular thinning compared with eyes unaffected by optic neuritis only in the outer temporal segment. Regarding the cohort with multiple sclerosis as a whole, nasal macular thinning is consistent with our peripapillary RNFL findings. Because the papillomacular bundle is located in the nasal macula, peripapillary RNFL thinning may result in a thinner macula secondary to loss of the RNFL in that region. Similar to our findings with SDOCT, Gugleta et al reported macular thinning in subjects with multiple sclerosis compared with controls, in addition to eyes 
Table 2 OR of an optic neuritis diagnosis as a function of RNFL and macular thickness measurements with the Heidelberg Spectralis ${ }^{\circledR}$ HRA + OCT in patients with multiple sclerosis and an OR of an optic neuritis diagnosis per $5 \mu \mathrm{m}$ decrease in RNFL or macular thickness

\begin{tabular}{llll}
\hline & Location & OR & $\boldsymbol{P}$ \\
\hline RNFL & Temporal & 1.33 & $\mathbf{0 . 0 0 8}$ \\
& Superior-temporal & 1.15 & $\mathbf{0 . 0 4}$ \\
& Superior-nasal & 1.04 & 0.37 \\
Nasal & 1.14 & 0.11 \\
& Inferior-nasal & 1.08 & 0.25 \\
& Inferior-temporal & 1.18 & $\mathbf{0 . 0 0 8}$ \\
& Superior & 1.12 & 0.08 \\
& Inferior & 1.16 & $\mathbf{0 . 0 4}$ \\
Macular Thickness & Average & 1.33 & $\mathbf{0 . 0 0 9}$ \\
& Inner temporal & 1.19 & 0.06 \\
& Inner superior & 1.15 & 0.08 \\
& Inner nasal & 1.10 & 0.22 \\
& Inner inferior & 1.16 & 0.10 \\
& Outer temporal & 1.08 & 0.50 \\
& Outer superior & 1.09 & 0.32 \\
& Outer nasal & 1.14 & 0.12 \\
& Outer inferior & 1.15 & 0.19 \\
& Central subfield & 1.11 & 0.18 \\
\hline
\end{tabular}

Abbreviations: OCT, optical coherence tomography; RNFL, retinal nerve fiber layer; OR, odds ratio.

affected or not affected by optic neuritis. ${ }^{31}$ Other investigators have demonstrated decreases in total macular volume in subjects with multiple sclerosis compared with controls, ${ }^{6,13,32}$ while decreases in macular volume in optic neuritis-affected eyes compared with optic neuritis-unaffected eyes have been reported as well. . $^{13,20,32,33}$

Although these earlier findings of macular volume abnormalities may be consistent with the concept of thinning secondary to axonal degeneration from multiple sclerosis and do not contradict our findings, the early reports, utilizing TDOCT exclusively, should be interpreted with caution. Calculation of total macular volume on a TDOCT device such as the Stratus ${ }^{\mathbb{B}}$ OCT requires a significant amount of interpolation because only six radial scans centered on the fovea are acquired. This is in contrast with potentially over 100 horizontal scans volumetrically acquired across the macula with SDOCT.

Multiple sclerosis is a progressive disease in which subclinical RNFL thinning may occur, even in patients who have not been clinically diagnosed with optic neuritis. ${ }^{15,16,18}$ In our study, we demonstrated progressive peripapillary RNFL thinning with increased duration of disease of more than five years, even in multiple sclerosis patients who do not manifest overt optic neuritis. Previously published reports using TDOCT have not consistently demonstrated such a relationship. ${ }^{6}$
In the current study, decreasing RNFL thickness was an excellent predictor of a previous diagnosis of optic neuritis in affected eyes (Table 2). This is evidenced by a significantly elevated risk of optic neuritis with peripapillary RNFL thinning. Given the retrospective nature of our study, larger prospective studies with SDOCT should be undertaken to define better an ideal RNFL thickness cutoff that is most likely to predict presence or absence of optic neuritis.

To our knowledge, our report represents one of the first such investigations of multiple sclerosis patients using highresolution SDOCT technology, given that most previously published reports utilized the older, much lower-resolution TDOCT. The difference between TDOCT and SDOCT is not trivial, ie, $400 \mathrm{~A}$-scans/sec versus 40,000 A-scans/sec, $10 \mu \mathrm{m}$ versus $5 \mu \mathrm{m}$ axial resolution, and six radial scans versus 100 to 145 horizontal scans for calculating macular volume for TDOCT versus SDOCT, respectively.

While previous investigators have demonstrated retinal changes using TDOCT in multiple sclerosis patients, the potential for improved precision, accuracy, point-by-point follow-up visit registration, and earlier detection of disease with SDOCT cannot be ignored. In addition to the technologic improvements listed above, SDOCT has other advantages, ie, true volumetric calculations (much less interpolation), image registration enabling accurate long-term monitoring, and improved image segmentation, facilitating analysis of specific retinal layers. Patients with multiple sclerosis, particularly those with subclinical eye disease (unaffected by optic neuritis), may have retinal microstructural changes that are imperceptible to the lower-resolution TDOCT.

A major challenge in utilizing SDOCT imaging is the abundance of machines on the market, each with distinct segmentation algorithms and normative databases. These issues must be taken into account when attempting to compare results obtained from different machines. While relatively few results have been reported with SDOCT technology in patients with multiple sclerosis, one group of researchers has reported high reliability of SDOCT for RNFL measurement in this setting. ${ }^{26}$ Bock et al demonstrated high correlation of RNFL measurements between a TDOCT and a SDOCT machine, but they also reported significant differences in corresponding measurements. ${ }^{25}$ Therefore, although some of the findings in our study parallel those of TDOCT-derived studies, it is best to make conclusions from data obtained from each machine separately because the absolute values of measurements cannot necessarily be compared between TDOCT and SDOCT, or even between two different SDOCT 
Table 3 Peripapillary RNFL and macular thinning with increasing duration of disease. Independent of age and a history of optic neuritis, multiple sclerosis patients with a longer duration of disease had significantly thinner peripapillary RNFL measurements. No such correlation was noted with respect to macular thickness measurements ( $\beta$ represents the correlation coefficient)

\begin{tabular}{|c|c|c|c|c|c|c|}
\hline RNFL & $\beta$ (13-60 months) & $95 \% \mathrm{Cl}$ & $P$ & $\beta$ ( $>60$ months $)$ & $95 \% \mathrm{Cl}$ & $P$ \\
\hline Temporal & -7.14 & $-16.43,2.16$ & 0.13 & -2.45 & $-11.03,6.13$ & 0.58 \\
\hline Superior-temporal & -19.67 & $-36.14,-3.20$ & 0.02 & -16.17 & $-31.68,-0.67$ & 0.04 \\
\hline Superior-nasal & -7.45 & $-24.20,9.30$ & 0.38 & -17.85 & $-32.37,-3.32$ & 0.02 \\
\hline Nasal & -0.35 & $-13.16,12.46$ & 0.96 & -10.82 & $-21.58,-0.06$ & 0.05 \\
\hline Inferior-nasal & 3.35 & $-12.01,18.70$ & 0.67 & -13.26 & $-29.96,3.44$ & 0.12 \\
\hline Inferior-temporal & -8.95 & $-23.56,5.67$ & 0.23 & -13.50 & $-25.68,-1.32$ & 0.03 \\
\hline Superior & -13.20 & $-27.11,0.7 \mid$ & 0.06 & -16.87 & $-30.03,-3.7 \mid$ & 0.01 \\
\hline Inferior & -3.00 & $-15.19,9.18$ & 0.63 & -13.49 & $-24.09,-2.90$ & 0.01 \\
\hline Average & -5.80 & $-15.69,4.08$ & 0.25 & -10.89 & $-18.97,-2.82$ & 0.008 \\
\hline Macular thickness & $\beta$ (13-60 months) & $95 \% \mathrm{Cl}$ & $P$ & $\beta$ ( $>60$ months) & $95 \% \mathrm{Cl}$ & $P$ \\
\hline Central subfield & -4.80 & $-|9.22,9.6|$ & $0.5 I$ & -4.80 & $-17.52,7.90$ & 0.46 \\
\hline Inner temporal & -5.58 & $-17.24,6.08$ & 0.35 & -7.18 & $-17.23,2.88$ & 0.16 \\
\hline Inner superior & -9.71 & $-22.72,3.31$ & 0.14 & -10.64 & $-22.19,0.90$ & 0.07 \\
\hline Inner nasal & -6.60 & $-19.75,6.46$ & 0.32 & -10.10 & $-21.08,0.84$ & 0.07 \\
\hline Inner inferior & -6.80 & $-20.01,6.33$ & 0.31 & -7.00 & $-\mid 9.01,5.01$ & 0.25 \\
\hline Outer temporal & -6.69 & $-18.16,4.77$ & 0.25 & -6.95 & $-16.97,3.08$ & 0.18 \\
\hline Outer superior & -8.59 & $-22.14,4.96$ & 0.21 & -10.13 & $-22.33,2.07$ & 0.10 \\
\hline Outer nasal & -6.79 & $-19.70,6.12$ & 0.30 & -9.09 & $-21.62,3.43$ & 0.16 \\
\hline Outer inferior & -7.92 & $-20.24,4.4 \mathrm{I}$ & 0.21 & -7.39 & $-19.27,4.50$ & 0.22 \\
\hline
\end{tabular}

Abbreviations: RNFL, retinal nerve fiber layer; $\mathrm{Cl}$, confidence interval.

machines. ${ }^{22,25,34}$ As an example, the Heidelberg software measures macular thickness from the internal limiting membrane to the bottom of the retinal pigment epithelium, the Topcon software measures from the internal limiting membrane to the top of the retinal pigment epithelium, and the Stratus measures from the internal limiting membrane to the photoreceptor inner segment/outer segment interface. Despite these challenges, future investigations utilizing imaging biomarkers for the monitoring of multiple sclerosis should utilize the more modern SDOCT rather than conventional TDOCT.

Future prospective investigations of multiple sclerosisrelated retinal changes should consider these findings when following subjects longitudinally. The retinal changes reported here need to be validated in multiple sclerosis disease subsets, and correlated with magnetic resonance imaging findings and measures of visual function, including visual acuity, contrast sensitivity, visual fields, microperimetry, vision-evoked potentials, and electroretinography. Nonetheless, as our findings demonstrate, SDOCT retinal imaging may represent a high-resolution, objective, noninvasive, and easily quantifiable in vivo biomarker of the presence of optic neuritis and severity of multiple sclerosis. It may also serve as a diagnostic adjunct for monitoring disease activity and response to treatment.

\section{Acknowledgment}

Research to Prevent Blindness provided support for this work.

\section{Disclosure}

The authors have no proprietary or financial interest in any aspect of this report.

\section{References}

1. Frohman EM, Fujimoto JG, Frohman TC, et al. Optical coherence tomography: A window into the mechanisms of multiple sclerosis. Nat Clin Pract Neurol. 2008;4:664-675.

2. Frisén L, Hoyt WF. Insidious atrophy of the retinal nerve fibers in multiple sclerosis. Fundoscopic identification in patients with and without visual complaints. Arch Ophthalmol. 1974;92:91-97.

3. Kerrison JB, Flynn T, Green WR. Retinal pathologic changes in multiple sclerosis. Retina. 1994;14:445-451.

4. Gordon-Lipkin E, Chodkowski B, Reich DS, et al. Retinal nerve fiber layer is associated with brain atrophy in multiple sclerosis. Neurology. 2007;69:1603-1609.

5. Grazioli E, Zivadinov R, Weinstock-Guttman B, et al. Retinal nerve fiber layer thickness is associated with brain MRI outcomes in multiple sclerosis. J Neurol Sci. 2008;268:12-17.

6. Henderson AP, Trip SA, Schlottmann PG, et al. An investigation of the retinal nerve fibre layer in progressive multiple sclerosis using optical coherence tomography. Brain. 2008;131:277-287.

7. Klistorner A, Arvind $\mathrm{H}$, Nguyen T, et al. Axonal loss and myelin in early ON loss in postacute optic neuritis. Ann Neurol. 2008;64:325-331.

8. Siger M, Dziegielewski K, Jasek L, et al. Optical coherence tomography in multiple sclerosis: Thickness of the retinal nerve fiber layer as a potential measure of axonal loss and brain atrophy. $J$ Neurol. 2008;255:1555-1560. 
9. Zaveri MS, Conger A, Salter A, et al. Retinal imaging by laser polarimetry and optical coherence tomography evidence of axonal degeneration in multiple sclerosis. Arch Neurol. 2008;65:924-928.

10. Albrecht P, Fröhlich R, Hartung HP, et al. Optical coherence tomography measures axonal loss in multiple sclerosis independently of optic neuritis. J Neurol. 2007;254:1595-1596.

11. Cheng H, Laron M, Schiffman JS, et al. The relationship between visual field and retinal nerve fiber layer measurements in patients with multiple sclerosis. Invest Ophthalmol Vis Sci. 2007;48:5798-5805.

12. Gundogan FC, Demirkaya S, Sobaci G. Is optical coherence tomography really a new biomarker candidate in multiple sclerosis - A structural and functional evaluation. Invest Ophthalmol Vis Sci. 2007;48: 5773-5781.

13. Pulicken M, Gordon-Lipkin E, Balcer LJ, et al. Optical coherence tomography and disease subtype in multiple sclerosis. Neurology. 2007; 69:2085-2092.

14. Sepulcre J, Murie-Fernandez M, Salinas-Alaman A, et al. Diagnostic accuracy of retinal abnormalities in predicting disease activity in MS Neurology. 2007;68:1488-1494.

15. Costello F, Hodge W, Pan YI, et al. Retinal nerve fiber layer and future risk of multiple sclerosis. Can J Neurol Sci. 2008;35:482-487.

16. Fisher JB, Jacobs DA, Markowitz CE, et al. Relation of visual function to retinal nerve fiber layer thickness in multiple sclerosis. Ophthalmology. 2006;113:324-332.

17. Noval S, Contreras I, Rebolleda G, Muñoz-Negrete FJ. Optical coherence tomography versus automated perimetry for follow-up of optic neuritis. Acta Ophthalmol Scand. 2006;84:790-794.

18. Parisi V, Manni G, Spadaro M, et al. Correlation between morphological and functional retinal impairment in multiple sclerosis patients. Invest Ophthalmol Vis Sci. 1999;40:2520-2527.

19. Costello F, Coupland S, Hodge W, et al. Quantifying axonal loss after optic neuritis with optical coherence tomography. Ann Neurol. 2006;59: 963-969.

20. Trip SA, Schlottmann PG, Jones SJ, et al. Retinal nerve fiber layer axonal loss and visual dysfunction in optic neuritis. Ann Neurol. 2005;58:383-391.

21. Costa-Cunha LV, Cunha LP, Malta RF, Monteiro ML. Comparison of Fourier-domain and time-domain optical coherence tomography in the detection of band atrophy of the optic nerve. Am J Ophthalmol. 2009; 147:56-63.

22. Knight OJ, Chang RT, Feuer WJ, Budenz DL. Comparison of retinal nerve fiber layer measurements using time domain and spectral domain optical coherent tomography. Ophthalmology. 2009;116:1271-1277.
23. Leung CK, Cheung CY, Weinreb RN, et al. Retinal nerve fiber layer imaging with spectral-domain optical coherence tomography: A variability and diagnostic performance study. Ophthalmology. 2009;116:1257-1263.

24. Sung KR, Kim DY, Park SB, Kook MS. Comparison of retinal nerve fiber layer thickness measured by Cirrus HD and Stratus optical coherence tomography. Ophthalmology. 2009;116:1264-1270.

25. Bock M, Brandt AU, Dörr J, et al. Time domain and spectral domain optical coherence tomography in multiple sclerosis: A comparative cross-sectional study. Mult Scler. 2010;16:893-896.

26. Syc SB, Warner CV, Hiremath GS, et al. Reproducibility of highresolution optical coherence tomography in multiple sclerosis. Mult Scler. 2010;16:829-839.

27. Grover S, Murthy RK, Brar VS, Chalam KV. Normative data for macular thickness by high-definition spectral-domain optical coherence tomography (Spectralis). Am J Ophthalmol. 2009;148:266-271.

28. Hube P. The behavior of maximum likelihood estimates under nonstandard conditions. Proceedings of the Fifth Berkeley Symposium on Mathematical Statistics and Probability. Vol 4. Lecom L, Neyman J, editor. Berkeley and Los Angeles; University of California Press; 1968.

29. Pan W. Akaike's information criterion in generalized estimating equations. Biometrics. 2001;57:120-125.

30. Kerrison JB, Flynn T, Green WR. Retinal pathologic changes in multiple sclerosis. Retina. 1994;14:445-451.

31. Gugleta K, Mehling M, Kochkorov A, et al. Pattern of macular thickness changes measured by ocular coherence tomography in patients with multiple sclerosis. Klin Monatsbl Augenheilkd. 2008;225:408-412.

32. Burkholder BM, Osborne B, Loguidice MJ, et al. Macular volume determined by optical coherence tomography as a measure of neuronal loss in multiple sclerosis. Arch Neurol. 2009;66:1366-1372.

33. Trip SA, Schlottmann PG, Jones SJ, et al. Optic nerve atrophy and retinal nerve fibre layer thinning following optic neuritis: Evidence that axonal loss is a substrate of MRI-detected atrophy. Neuroimage. 2006;31:286-293.

34. Johnson DE, El-Defrawy SR, Almeida DR, Campbell RJ. Comparison of retinal nerve fibre layer measurements from time domain and spectral domain optical coherence tomography systems. Can J Ophthalmol. 2009;44:562-566
Clinical Ophthalmology

\section{Publish your work in this journal}

Clinical Ophthalmology is an international, peer-reviewed journal covering all subspecialties within ophthalmology. Key topics include: Optometry; Visual science; Pharmacology and drug therapy in eye diseases; Basic Sciences; Primary and Secondary eye care; Patient Safety and Quality of Care Improvements. This journal is indexed on

\section{Dovepress}

PubMed Central and CAS, and is the official journal of The Society of Clinical Ophthalmology (SCO). The manuscript management system is completely online and includes a very quick and fair peer-review system, which is all easy to use. Visit http://www.dovepress.com/ testimonials.php to read real quotes from published authors. 